\title{
Solution of the radiative transfer problems in two-dimensional participating cylindrical medium with isotropic scattering using the SKN approximation
}

\author{
N. Döner ${ }^{1} \&$ Z. Altaç ${ }^{2}$ \\ ${ }^{I}$ Mechanical Engineering Department, Dumlupınar University, \\ Kütahya, Turkey \\ ${ }^{2}$ Eskişehir Osmangazi University, Eskişehir, Turkey
}

\begin{abstract}
The $S K_{N}$ (Synthetic Kernel) approximation is applied to a two-dimensional homogeneous cylindrical participating medium with isotropic scattering. The $S K_{N}$ equations are tested against benchmark problems consisting of cold homogeneous participating medium. The solutions are compared with those obtained by various methods available in the literature. The $S K_{3}$ approximation results for geometries of very large and very small aspect ratios are in excellent agreement with those of benchmark solutions. The $S K_{3}$ solutions with moderate aspect ratios are accurate within several percent.

Keywords: synthetic kernel method, participating medium, isotropic scattering, two-dimensional cylindrical medium.
\end{abstract}

\section{Introduction}

The $S K_{N}$ approximation which was developed to solve neutron transport equation can also be applied to radiative integral transfer equation (RITE) [1]. The significance of solving the integral equations is that one has to deal with the spatial variables rather than spatial and angular variables that exist in radiative transfer equation (RTE)-Boltzmann's equation. Thus the solutions obtained from the integral equations do not exhibit ray effect which is a phenomena that plagues discrete ordinates and similar methods. Ray effect is the result of discretization of angular variables. 
The $S K_{N}$ method is applied to the RITE by substituting approximations to its radiative transfer kernels through a sum of exponentials, as in the exponential kernel approximation. Then RITE can be reduced into a set of coupled second-order partial differential equations, which will be referred to as the $S K_{N}$ equations. Physical boundary conditions are embedded in the force functions (surface integrals) of the RITE which are exactly preserved in the $S K_{N}$ equations; however, boundary conditions (BCs) of mathematical in nature are required to solve the $S K_{N}$ equations.

Recently, the applicability and validity of the $S K_{N}$ approximation to thermal radiative transfer problems of one dimensional plane parallel and spherical geometries, and two-dimensional participating homogeneous and inhomogeneous medium have been investigated [2-4]. Solutions obtained with the $S K_{N}$ method are very accurate and certainly superior to $\mathrm{P}_{1}$ and differential or modified differential approximations. It has been also demonstrated that in twodimensional geometries the $S K_{N}$ method is a high order approximation which contains no ray effect [4]. Solving second order elliptic differential equations with simple boundary conditions lead to less computational efforts and cpu time.

In this study, the $S K_{N}$ method with Gauss quadrature set has been applied to radiative transfer in two-dimensional cylindrical participating homogeneous medium. The accuracy and convergence of the method are investigated.

\section{Derivations of $S K_{N}$ equations}

The $S K_{N}$ equations for two-dimensional cylindrical medium can written as [9]

$$
\left\{-\mu_{n}^{2}\left(\frac{\partial^{2}}{\partial \tau_{r}^{2}}+\frac{1}{\tau_{r}} \frac{\partial}{\partial \tau_{r}}+\frac{\partial^{2}}{\partial \tau_{z}^{2}}\right)+1\right\} G_{n}\left(\tau_{r}, \tau_{z}\right)=S\left(\tau_{r}, \tau_{z}\right), \quad n=1,2, \ldots, N
$$

where $\left(\tau_{r}, \tau_{z}\right)$ are the optical coordinates, $G_{n}\left(\tau_{r}, \tau_{z}\right)$ is a function representing the $n$th component of the synthetic kernel function, $S\left(\tau_{r}, \tau_{z}\right)$ is the isotropic source function for a cold medium and is defined as

$$
S\left(\tau_{r}, \tau_{z}\right)=\omega G\left(\tau_{r}, \tau_{z}\right)
$$

and $\omega$ is the scattering albedo. The incident radiation and heat flux can be defined as

$$
\begin{gathered}
G\left(\tau_{r}, \tau_{z}\right)=F_{1}\left(\tau_{r}, \tau_{z}\right)+\sum_{n=1}^{N} w_{n} G_{n}\left(\tau_{r}, \tau_{z}\right) \\
\mathbf{q}\left(\tau_{r}, \tau_{z}\right)=\mathbf{F}_{2}\left(\tau_{r}, \tau_{z}\right)+\sum_{n=1}^{N} w_{n} \mathbf{q}_{n}\left(\tau_{r}, \tau_{z}\right)
\end{gathered}
$$

where $\left(\mu_{n}, w_{n}\right)$ are the Gauss quadratures for $\mu \in(0,1), \mathbf{q}_{n}\left(\tau_{r}, \tau_{z}\right)$ is a vector function defined as $\mathbf{q}_{n}\left(\tau_{r}, \tau_{z}\right)=-\mu_{n}^{2} \nabla G_{n}\left(\tau_{r}, \tau_{z}\right)$ [4], $F_{1}\left(\tau_{r}, \tau_{z}\right)$ and $\mathbf{F}_{2}\left(\tau_{r}, \tau_{z}\right)$ 
are the force functions containing physical boundary conditions (BCs). Then the net heat flux components can be also be expressed as

$$
\begin{aligned}
& q_{r}\left(\tau_{r}, \tau_{z}\right)=F_{2 r}\left(\tau_{r}, \tau_{z}\right)-\sum_{n=1}^{N} w_{n} \mu_{n}^{2} \frac{\partial G_{n}\left(\tau_{r}, \tau_{z}\right)}{\partial \tau_{r}} \\
& q_{z}\left(\tau_{r}, \tau_{z}\right)=F_{2 z}\left(\tau_{r}, \tau_{z}\right)-\sum_{n=1}^{N} w_{n} \mu_{n}^{2} \frac{\partial G_{n}\left(\tau_{r}, \tau_{z}\right)}{\partial \tau_{z}}
\end{aligned}
$$

The $S K_{N}$ equations are subject to the following mathematical BCs regardless of the physical BCs:

$$
\begin{array}{cc}
\text { For } \tau_{r}=0, & \frac{\partial G_{n}\left(0, \tau_{z}\right)}{\partial \tau_{r}}=0 \\
\text { For } \tau_{z}=0, & \frac{\partial G_{n}\left(\tau_{r}, 0\right)}{\partial \tau_{z}}=\frac{1}{\mu_{n}} G_{n}\left(\tau_{r}, 0\right) \\
\text { For } \tau_{z}=L, & \frac{\partial G_{n}\left(\tau_{r}, L\right)}{\partial \tau_{z}}=-\frac{1}{\mu_{n}} G_{n}\left(\tau_{r}, L\right) \\
\text { For } \tau_{r}=R, & \frac{\partial G_{n}\left(R, \tau_{z}\right)}{\partial \tau_{r}}=-\frac{K_{1}\left(R / \mu_{n}\right)}{\mu_{n} K_{0}\left(R / \mu_{n}\right)} G_{n}\left(R, \tau_{z}\right)
\end{array}
$$

where $K_{0}(x)$ and $K_{1}(x)$ are zeroth and first order modified Bessel functions. These BCs are adapted from the exact boundary conditions of the onedimensional $S K_{N}$ derivations.

The source term of the $S K_{N}$ equations is given by Eq. (2). If the medium is pure absorber, Eq. (1) yields zero solution for $G_{n}$. Then the RITE is no longer an integral equation and the solutions for the incident energy and the net radiative heat flux; respectively, are simply $F_{1}\left(\tau_{r}, \tau_{z}\right)$ and $\mathbf{F}_{2}\left(\tau_{r}, \tau_{z}\right)$ which are the exact solutions. As the scattering albedo increases, source coupling becomes stronger. If an iterative scheme is used in the numerical solution of the $S K_{N}$ equations, computation time for pure scattering cases is the highest. Previous studies revealed that the errors in the $S K_{N}$ method are also the highest for the pure scattering medium [2-4].

\section{Results and discussions}

In order to test the applicability and the accuracy of the $S K_{N}$ approximation, we have adapted the following benchmark problems [9]:

Benchmark Problem 1 (BP-1): A Short cylindrical medium with large optical radius, aspect ratios of 10 to 20 , can be considered as a plane parallel geometry. The medium is homogeneous, cold and pure scattering $(\omega=1)$. The two-dimensional, solid cylinder solutions of $S K_{N}$ method for a combination of 
optical radius of 0.2 to 25 mean free path ( $\mathrm{mfp}$ ) and height of 0.002 to $5 \mathrm{mfp}$ are compared with the plane parallel geometry solutions of Crosbie and Viskanta [5] for the incident energy at the top and the incident energy and the net radiative heat flux at bottom.

Benchmark Problem 2 (BP-2): Long cylindrical geometry with small aspect rations for a cold medium with scattering albedos of $\omega=0.3,0.5$ and 0.9 are considered. The two dimensional cylindrical $S K_{N}$ solutions for the incident energy at the center and the incident energy and the net radiative heat flux at the surface are compared with the one-dimensional cylindrical RITE solutions [6].

Benchmark Problem 3 (BP-3): Radiative transfer in a two-dimensional cylindrical medium (Figure 1) which is subject to collimated unit irradiation on top surface was considered [7]. The other surfaces are cold and transparent. The medium is also cold, homogeneous with pure isotropic scattering $(\omega=1)$. The incident energy and net radiative heat flux solutions using $S K_{N}$ approximation are compared with those of $\mathrm{Wu}$ and $\mathrm{Wu}$ [7] and Hsu et al. [8].

For BP-3, the surface integrals of the incident energy and the net radiative heat flux in the RITE yield [7]:

$$
\begin{gathered}
F_{1}\left(\tau_{r}, \tau_{z}\right)=\exp \left(-\tau_{z}\right) \\
F_{2 r}\left(\tau_{r}, \tau_{z}\right)=0 \\
F_{2 z}\left(\tau_{r}, \tau_{z}\right)=\exp \left(-\tau_{z}\right)
\end{gathered}
$$

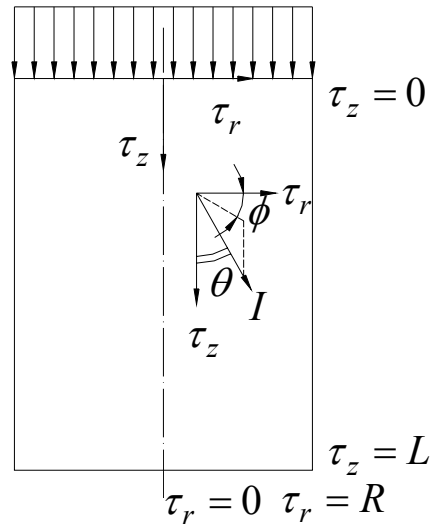

Figure 1: The geometry and the coordinate system.

This study was carried out on a Pentium III $800 \mathrm{MHz}$ processor with $512 \mathrm{Mb}$ RAM. Computation time with $S K_{1}, S K_{2}$ and $S K_{3}$ approximations naturally increase with the order of approximation since the number of differential equations to be solved are also increased.

In Table 1, the effects of grid refinement and CPU time on numerical calculations are depicted. It is clear that as the scattering albedo is increased for a 
fixed grid size and the same order of approximation, cpu time increases due to strong coupling in the $S K_{N}$ equations which requires more iterations for convergence. However, $S K_{N}$ equations can be solved directly using a block tri-diagonal solver in which case the CPU time remains nearly the same. Several cases of grid combinations were considered to ensure grid independence of the presented solutions within four significant digits. The grid structure was increased with the optical dimensions such as using $M_{r} \times M_{z}=50 \times 100$, $M_{r} \times M_{z}=100 \times 200$ and $M_{r} \times M_{z}=200 \times 200$ for cylinders with $R=0.125$ and $L=0.25 \mathrm{mfp}, R=0.5$ and $L=1.0 \mathrm{mfp}$, and $R=2.0$ and $L=4.0 \mathrm{mfp}$, respectively.

Table 1: The effect of cpu time and grid refinement for cylinder of $R=0.125 \mathrm{mfp}$ and $L=0.25 \mathrm{mfp}$.

\begin{tabular}{|c|c|c|c|c|c|c|}
\hline \multirow{3}{*}{$M_{r} \times M_{z}$} & \multicolumn{3}{|c|}{$\omega=0.5$} & \multicolumn{3}{|c|}{$\omega=1.0$} \\
\hline & $S K_{l}$ & $S K_{2}$ & $S K_{3}$ & $S K_{l}$ & $S K_{2}$ & $\mathrm{SK}_{3}$ \\
\hline & \multicolumn{3}{|c|}{ CPU (sec) } & \multicolumn{3}{|c|}{ CPU (sec) } \\
\hline $10 \times 10$ & 0.016 & 0.034 & 0.062 & 0.189 & 0.262 & 0.412 \\
\hline $10 \times 20$ & 0.184 & 0.635 & 0.671 & 0.283 & 0.431 & 0.876 \\
\hline $25 \times 50$ & 1.414 & 2.765 & 4.215 & 2.084 & 4.814 & 8.0 \\
\hline $50 \times 50$ & 4.75 & 9.69 & 31.85 & 9.26 & 20.98 & 39.12 \\
\hline $50 \times 100$ & 33.7 & 72.5 & 149.3 & 68.6 & 173.9 & 432.0 \\
\hline
\end{tabular}

Table 2: $\quad$ Comparisons of the solutions for BP-1.

\begin{tabular}{|c|c|c|c|c|c|c|c|c|c|c|c|}
\hline \multicolumn{3}{|c|}{$\omega=1.0$} & \multicolumn{3}{|c|}{ Exact (Ref. [5]) } & \multirow{2}{*}{\multicolumn{3}{|c|}{\begin{tabular}{|c|}
$\boldsymbol{S K}_{\mathbf{2}}$ \\
$G(0,0) G(0, L) q(0, L)$
\end{tabular}}} & \multicolumn{3}{|c|}{$\mathrm{SK}_{3}$} \\
\hline $\boldsymbol{R}$ & $L$ & $\gamma$ & $G(0)$ & $G(L)$ & $q(L)$ & & & & $G(0,0)$ & $G(0,1$ & $a(0, L)$ \\
\hline 0.2 & & 20 & & & & & 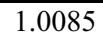 & & & & \\
\hline 2 & 0. & 20 & & & & 1.24 & 1.06 & & & & \\
\hline 5 & 0.5 & 20 & 8 & 1.0538 & 5 & 1 . & 1.06 & 74 & & & 976 \\
\hline 5 & 1 & 10 & & & & & 0.9 & 6 & & & \\
\hline 10 & 2 & 10 & 2. & 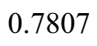 & 5 & 6 & 0.7 & 29 & 1 & & 34 \\
\hline 12 & 2.5 & 10 & 27 & 8 & 0.42 & 2.17 & 0.70 & 0.42 & & & 0.4252 \\
\hline 25 & 5.0 & 10 & 4552 & 4504 & 0.2613 & 2.46 & 0.45 & 0.2 & 2.4 & 0.45 & 0.2 \\
\hline
\end{tabular}

In Table 2, the relative errors of $G(0,0), G(0, L)$ and $q(0, L)$ for BP-1 are compared with the exact plane parallel solutions [5]. The agreement of the exact and the $S K_{N}$ solutions, with increasing orders, is excellent. For example, the $S K_{3}$ solutions for pure scattering medium $(\gamma=20$ and $L=0.5 \mathrm{mfp})$ yield relative errors of $-0.26 \%,-0.32 \%$ and $-0.01 \%$ for $G(0,0), G(0, L)$ and $q(0, L)$, respectively. The errors for the same cylinder are $0.07 \%, 0.10 \%$ and $0.03 \%$ in-though not given in Table 2-Wu and $\mathrm{Wu}$ [7]'s study. For $\gamma=10$ and $L=1 \mathrm{mfp}$, the errors for $G(0,0), G(0, L)$ and $q(0, L)$ are $0.03 \%, 0.27 \%$ and $-0.12 \%$, respectively, when using $\mathrm{SK}_{3}$ approximation. In Wu and Wu [7]'s study, the errors are found to be $0.28 \%, 0.52 \%$ and $0.23 \%$, respectively. In other cylinder configurations, the errors when using the $S K_{3}$ approximation have the same order of magnitude. 
Comparisons of the exact solutions [6], of BP-2 with the SKN solutions are given in Table 3 for various scattering albedos. For a medium with $\omega=0.9$ and $R=0.5 \mathrm{mfp}$, the relative errors of $G(0), G(R)$ and $q(R)$ with $S K_{3}$ approximation are found to be $-0.99 \%, 0 \%$ and $-0.63 \%$, respectively. For the same scattering albedo and $R=1.0 \mathrm{mfp}$, the errors yield $0.50 \%, 0.02 \%$ and $-0.67 \%$ and for $R=2.5 \mathrm{mfp},-0.15 \%,-0.19 \%$ and $-1.77 \%$ for $G(0), G(R)$ and $q(R)$, respectively. The $S K_{2}$ solutions are in excellent agreement yielding 2 to 3 significant digit accuracies, however, as the medium becomes strongly scattering, higher order, such as $S K_{3}$ approximation, becomes necessary.

Table 3: $\quad$ Comparisons of the solutions for Benchmark Problem 2.

\begin{tabular}{|c|c|c|c|c|c|c|c|c|c|}
\hline \multicolumn{2}{|r|}{$R=0.5$} & \multicolumn{8}{|l|}{$L=5$} \\
\hline$\omega$ & \multicolumn{2}{|c|}{$G(0) / 4 \pi G(R) / 4 \pi$} & $-q(R)$ & \multicolumn{3}{|c|}{$G(0) / 4 \pi G(R) / 4 \pi-q(R)$} & \multicolumn{3}{|c|}{$G(0) / 4 \pi G(R) / 4 \pi-q(R)$} \\
\hline 0.3 & 0.6011 & 0.7891 & 1.4890 & 0.6059 & 0.7891 & 1.4886 & 0.6026 & 0.7889 & 1.4900 \\
\hline 0.5 & 0.6828 & 0.8348 & 1.1707 & 0.6917 & 0.8352 & 1.1704 & 0.6858 & 0.8346 & 1.1723 \\
\hline 0.9 & 0.9183 & 0.9588 & 0.2939 & 0.9388 & 0.9609 & 0.2947 & 0.9274 & 0.9589 & 0.2957 \\
\hline \multicolumn{2}{|r|}{$R=1.0$} & $L=5$ & & & & & & & \\
\hline 0.3 & 0.3644 & 0.6944 & 2.1647 & 0.3658 & 0.6937 & 2.1666 & 0.3636 & 0.6943 & 2.1660 \\
\hline 0.5 & 0.4571 & 0.7475 & 1.8014 & 0.4608 & 0.7465 & 1.8054 & 0.4554 & 0.7474 & 1.8036 \\
\hline 0.9 & 0.8247 & 0.9249 & 0.5453 & 0.8440 & 0.9246 & 0.5523 & 0.8205 & 0.9247 & 0.5490 \\
\hline 0.3 & 0.0811 & 0.6030 & 2.7308 & 0.0807 & 0.6028 & 2.7370 & 0.0812 & 0.6035 & 2.7384 \\
\hline 0.5 & 0.1266 & 0.6510 & 2.4317 & 0.1254 & 0.6507 & 2.4421 & 0.1268 & 0.6518 & 2.4439 \\
\hline 0.9 & 0.5298 & 0.8548 & 1.0522 & 0.5238 & 0.8547 & 1.0713 & 0.5306 & 0.8565 & 1.0709 \\
\hline
\end{tabular}

In Figure 2, the radial distribution of the incident radiation at the top and bottom surfaces of BP-3 are given comparatively with those of Refs [7] and [8] for cylinder with optical dimensions of $R=0.125$ and $L=0.25 \mathrm{mfp}$. The maximum relative errors with $S K_{3}$ approximation on the top surface are $-3.06 \%$ and $-1.87 \%$ in comparison to the solutions of [7] and [8], respectively. On the other hand, these errors with $S K_{3}$ approximation on the bottom surface are $-2.4 \%$ and $2.1 \%$ in comparison to the solutions of [7] and [8], respectively.

In Figure 3, the axial distribution of the incident radiation at the center and outer surface are depicted comparatively with those of [7] and [8] for cylinder with optical dimensions of $R=0.125$ and $L=0.25 \mathrm{mfp}$. The maximum relative errors with $\mathrm{SK}_{3}$ approximation at the centerline are $-2.4 \%$ and $-2.06 \%$ in comparison to the solutions of [7] and [8], respectively; whereas, these errors for the outer surface are $-2.32 \%$ and $-1.92 \%$.

In Figure 4, the radial distributions of the incident radiation at the top and bottom surfaces are comparatively presented with those of [7] and [8] for cylinder with optical dimensions of $R=0.5$ and $L=1.0 \mathrm{mfp}$. The maximum relative errors with $S K_{3}$ approximation on the top surface are $-5.36 \%$ and $3.96 \%$ in comparison to [7] and [8] solutions, respectively; while the maximum errors with $\mathrm{SK}_{3}$ approximation on the bottom surface are $-8.5 \%$ and $-3.9 \%$ when compared to those of [7] and [8] solutions, respectively. 


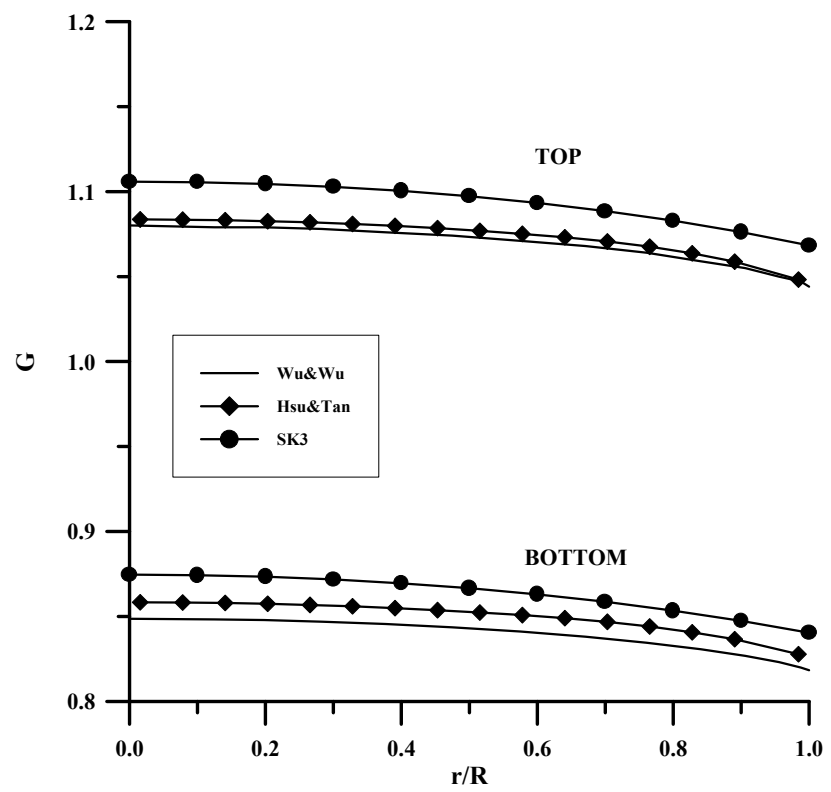

Figure 2: Radial distribution of the incident radiation at the top and bottom surfaces for cylinder with optical dimensions of $R=0.125 \mathrm{mfp}$ and $L=0.25 \mathrm{mfp}$.

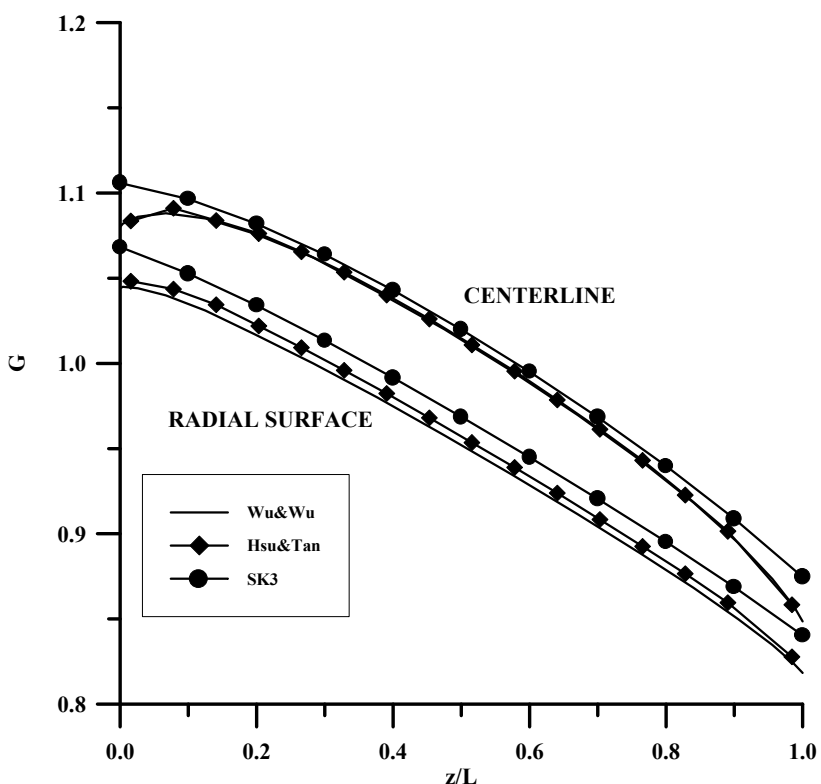

Figure 3: Axial distribution of the incident radiation at the center and outer surface for cylinder with optical dimensions of $R=0.125 \mathrm{mfp}$ and $L=0.25 \mathrm{mfp}$. 


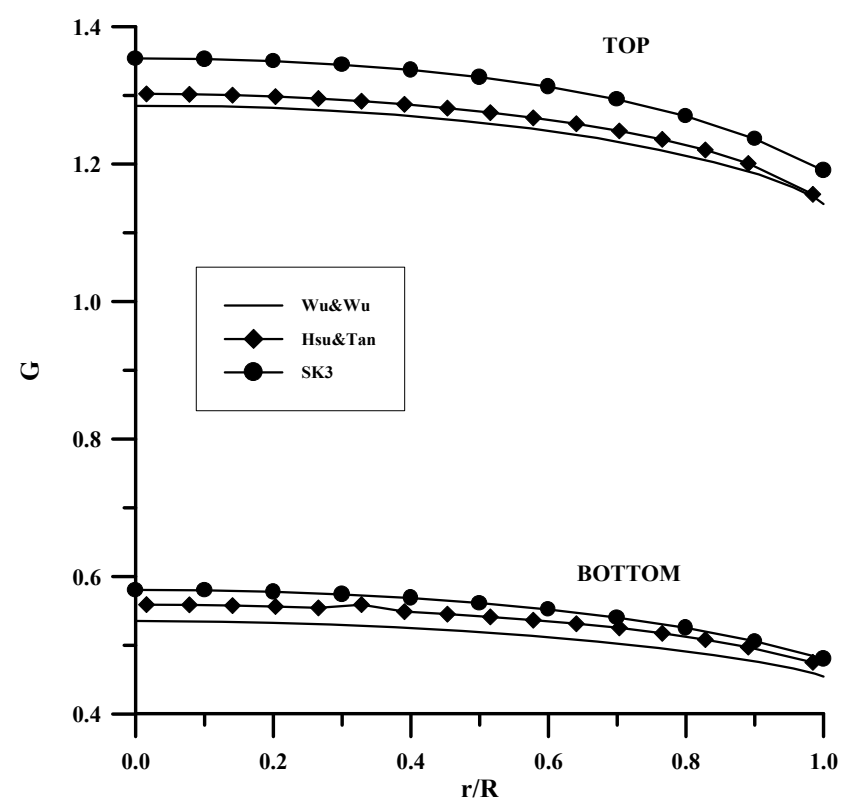

Figure 4: Radial distribution of the incident radiation at the top and bottom Surfaces cylinder with optical dimensions of $R=0.5 \mathrm{mfp}$ and $L=1 \mathrm{mfp}$.

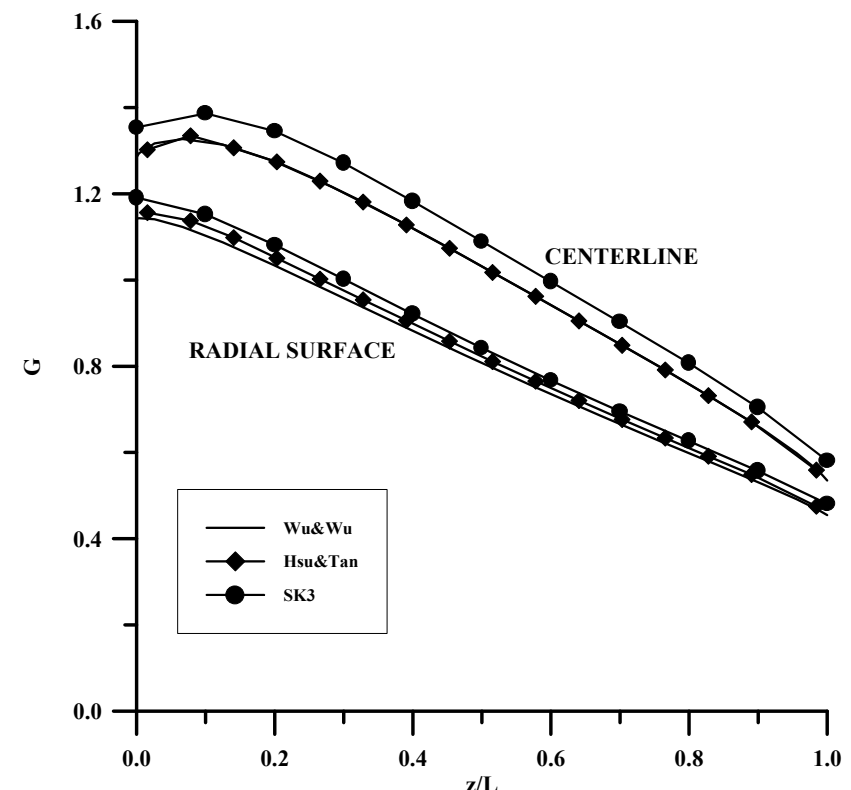

Figure 5: Axial distribution of the incident radiation at the center and outer surface for cylinder with optical dimensions of $R=0.5 \mathrm{mfp}$ and $L=1 \mathrm{mfp}$. 
In Figure 5, the axial distribution of the incident radiation at the center and outer surface using $S K_{3}$ approximation are shown comparatively with those of [7] and [8] for cylinder with $R=0.5$ and $L=1.0 \mathrm{mfp}$. The maximum relative errors with $S K_{3}$ are $-5.4 \%$ and $-3.97 \%$ at the center and $-4.3 \%$ and $-3.0 \%$ at the outer surface with respect to the solutions of [7] and [8], respectively.

In Figure 6, the radial distributions of the incident radiation using SK3 approximation at the top and bottom surfaces are comparatively depicted with those of [7] and [8] for $R=2$ and $L=4 \mathrm{mfp}$ cylinder. The maximum relative errors of $S K_{3}$ approximation on the top surface are $-3.03 \%$ and $3.14 \%$ in comparison to the solutions of [7] and [8], respectively. On the other hand, the maximum errors with $S K_{3}$ approximation on the bottom surface are $-8.5 \%$ and $8.5 \%$ with respect to those of [7] and [8], respectively.

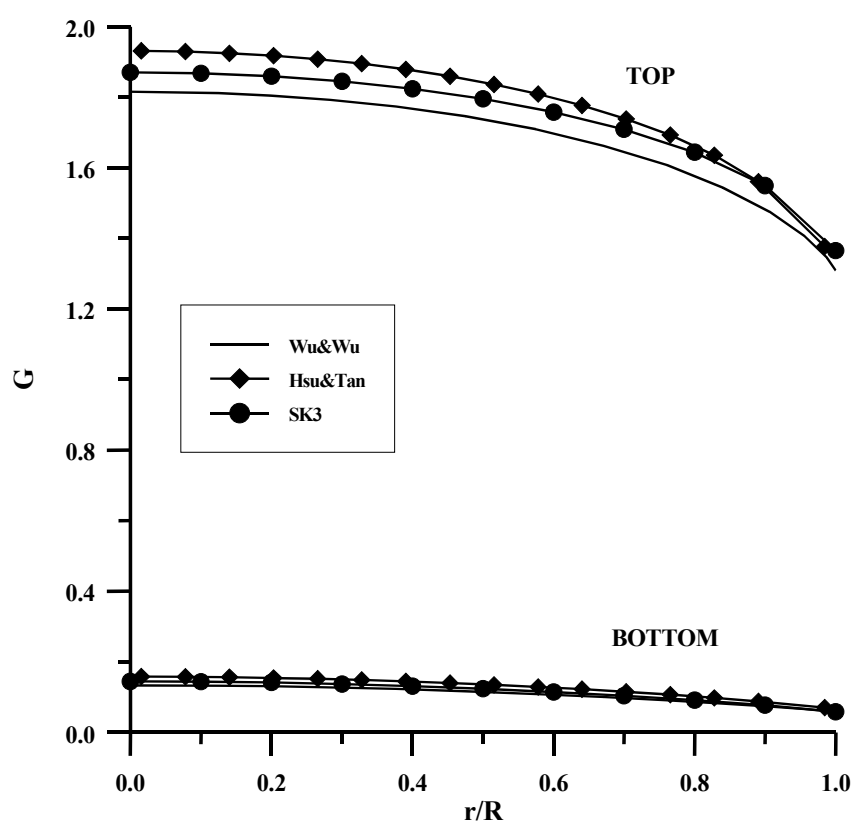

Figure 6: Radial distribution of the incident radiation at the top and bottom surfaces for cylinder with optical dimensions of $R=2 \mathrm{mfp}$ and $L=4 \mathrm{mfp}$.

In Figure 7, the axial distribution of the incident radiation using $S K_{3}$ approximation at the center and outer surface are depicted comparatively with those of [7] and [8] for cylinder with $R=2$ and $L=4 \mathrm{mfp}$. The maximum relative errors with $S_{3}$ approximation at the centerline are $-3.03 \%$ and $3.14 \%$ in comparison to the solutions of [7] and [8] respectively; whereas, these errors for the outer surface are $-4.31 \%$ and $0.83 \%$, respectively. 
118 Advanced Computational Methods in Heat Transfer IX

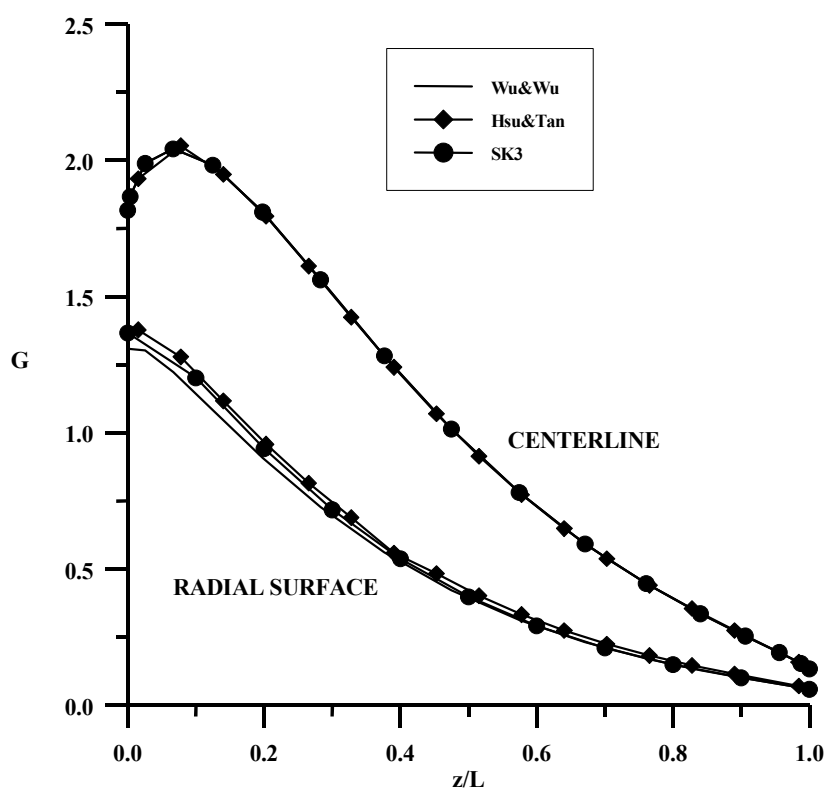

Figure 7: Axial distribution of the incident radiation at the center and outer surface for cylinder with optical dimensions of $R=2 \mathrm{mfp}$ and $L=4 \mathrm{mfp}$.

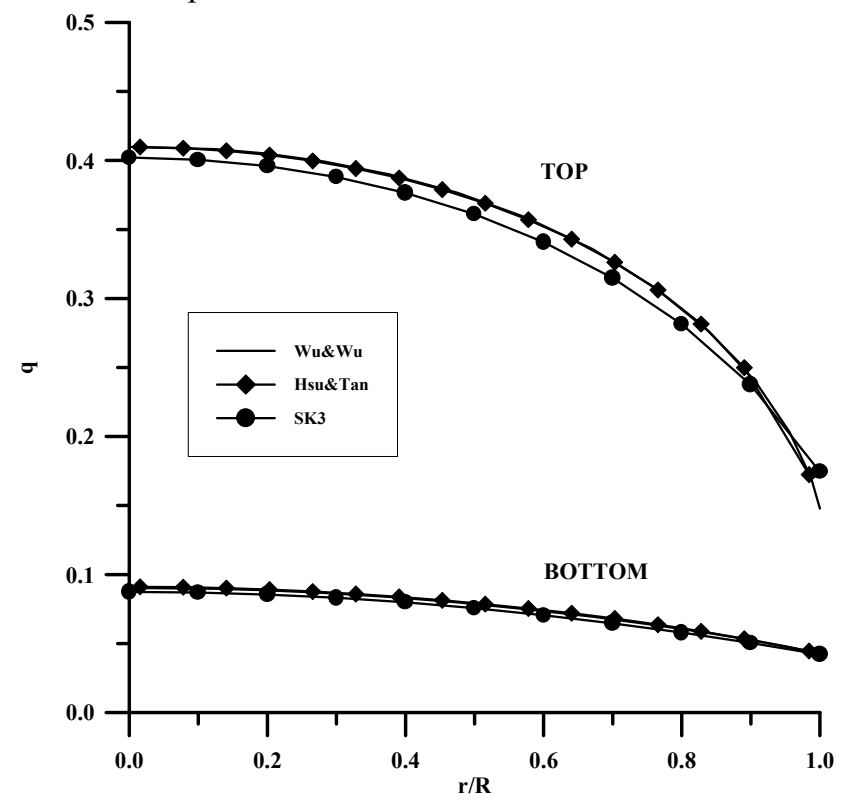

Figure 8: Radial distribution of the radiative heat flux at the top and bottom surfaces for cylinder with optical dimensions of $R=2 \mathrm{mfp}$ and $L=4 \mathrm{mfp}$. 
The incoming and/or outgoing radiative heat fluxes using $S K_{3}$ also compare well within several percent errors, generally yielding underestimated values, with the referred studies. To illustrate the behavior of the radiative heat fluxes, we considered the distribution of cylinder with optical dimensions of $R=2$ and $L=4$ $\mathrm{mfp}$. In Figure 8, the radial distribution of the outgoing radiative heat flux using $\mathrm{SK}_{3}$ approximation at the top and bottom surfaces are comparatively depicted with those of [7] and [8]. Solutions at the top and bottom surfaces from $\mathrm{Wu}$ and $\mathrm{Wu}$ [7] and Hsu et al. [8] almost coincide, and thus two the lines are not distinguishable. The maximum relative errors with $S K_{3}$ approximation on the bottom surface are $-1.92 \%$ and $-1.87 \%$ in comparison to those of [7] and [8]], respectively, while these relative errors are -3.21 and $-3.98 \%$, respectively.

\section{Conclusions}

The $S K_{N}$ approximation solutions for various two-dimensional homogeneous cylindrical mediums with isotropic scattering are compared with the solutions available in the literature. This study concludes the following: (i) the method is very accurate yielding 2-3 significant accurate solutions for mostly absorbing medium while the highest errors occur in pure scattering medium, (ii) the $S K_{N}$ equations can be numerically solved very easily with no numerical complexities, (iii) the approximation requires much less then computational effort when compared to the cpu time requirements of the exact RITE of the same grid configuration, (iv) the approximation can be improved especially at low orders by the selection of the synthetic kernel quadratures, (v) the method for moderate aspect ratios yield relative errors of several percent for pure scattering medium; however, medium with some absorption yield less errors, (vi) the method is free of "ray effect".

\section{References}

[1] Altaç Z. and Spinrad B.I., The $\mathrm{SK}_{\mathrm{N}}$ method I: A high order transport approximation to neutron transport problems, Nuclear Science and Engineering, 106, pp. 471-479,1990.

[2] Altaç Z., The $S K_{N}$ approximation for solving radiative transfer problems in absorbing, emitting, and isotropically scattering plane-parallel medium: part 1, ASME Journal of Heat Transfer, 124, pp. 674-684, 2002 a.

[3] Altaç Z., The $S K_{N}$ approximation for solving radiative transfer problems in absorbing, emitting, and linear anisotropically scattering plane-parallel medium: part 2, ASME Journal of Heat Transfer, 124, pp. 685-695, 2002 b.

[4] Altaç Z. and Tekkalmaz M., The $S K_{N}$ approximation for solving radiation transport problems in absorbing, emitting and scattering rectangular geometries, JQSRT, Volume 73, No. 4, pp. 219-230, 2002.

[5] Crosbie A.L. and Viskanta R., Nongray Radiative Transfer in a Planar Medium Exposed to a Collimated Flux, JQSRT, Volume 10, pp. 465-485, 1970. 
[6] Altaç Z., Radiative Transfer in Absorbing, Emitting and Linearly Anisotropic-Scattering Inhomogeneous Cylindrical Medium, JQSRT, Volume 77, pp. 177-192, 2003.

[7] Wu S.C. and Wu C.Y., Radiative Heat Transfer in a Two-Dimensional Cylindrical Medium Exposed to Collimated Radiation, International Comm. Heat \& Mass Transfer, Volume24, No 4, pp. 475-484, 1997.

[8] Hsu P.F., Tan Z.M., Wu S.H. and Wu C.Y., Radiative Heat Transfer in Finite Cylindrical Homogeneous and Nonhomogeneous Scattering Media Exposed to Collimated Radiation, Numerical Heat Transfer, Part A, Volume35, pp. 655-679, 1999.

[9] Döner N., Calculation of Radiative Heat Transfer in Cylindrical Participating Medium Using the $S K_{N}$ Method, Ph. D. Thesis, Osmangazi University (in Turkish), 2003. 